\title{
Interaction of Single Viruslike Particles with Vesicles Containing Glycosphingolipids
}

\author{
M. Bally, ${ }^{1}$ A. Gunnarsson, ${ }^{1}$ L. Svensson, ${ }^{2}$ G. Larson, ${ }^{3}$ V. P. Zhdanov, ${ }^{1,4}$ and F. Höök ${ }^{1, *}$ \\ ${ }^{1}$ Department of Applied Physics, Chalmers University of Technology, S-412 96 Göteborg, Sweden \\ ${ }^{2}$ Division of Molecular Virology, University of Linköping, Linköping, Sweden \\ ${ }^{3}$ Department of Clinical Chemistry and Transfusion Medicine, Sahlgrenska Academy, University of Gothenburg, Göteborg, Sweden \\ ${ }^{4}$ Boreskov Institute of Catalysis, Russian Academy of Sciences, Novosibirsk 630090, Russia
}

(Received 11 April 2011; published 28 October 2011)

\begin{abstract}
Glycosphingolipids are involved in the first steps of virus-cell interaction, where they mediate specific recognition of the host cell membrane. We have employed total-internal-reflection fluorescence microscopy to explore the interaction kinetics between individual unlabeled noroviruslike particles, which are attached to a glycosphingolipid-containing lipid bilayer, and fluorescent vesicles containing different types and concentrations of glycosphingolipids. Under association equilibrium, the vesicle-binding rate is found to be kinetically limited, yielding information on the corresponding activation energy. The dissociation kinetics are logarithmic over a wide range of time. The latter is explained by the vesiclesize-related distribution of the dissociation activation energy. The biological, pharmaceutical, and diagnostic relevance of the study is briefly discussed.
\end{abstract}

DOI: 10.1103/PhysRevLett.107.188103

PACS numbers: 87.15.kp, 87.15.Fh, 87.19.xd

Viruses consist of DNA or RNA genomes protected by a protein capsid or a lipidic membrane envelope. Their replication cycles include virion attachment to a host cell, penetration, uncoating and release of genome, genome replication, viral protein synthesis, capsid assembly, and escape from the host [1]. An in-depth understanding of these processes plays a key role for development of antiviral therapies, vaccines, and high-performance diagnostics. Huge efforts are therefore undertaken to develop assays capable of elucidating virus-cell interactions with high sensitivity. Recently, techniques have emerged [2,3] that enable real-time optical visualization of single virions in live cells. This represents an important progress over conventional transmission electron microscopy, as these techniques make it possible to quantify the dynamics of the infectious entry pathway. Commonly used cell-based techniques are complemented with in vitro bioanalytical assays employing model surface-supported cell membranes of chosen composition [4-6]. Such studies, focused on, e.g., ensemble-averaged recording of binding to modelsupported lipid bilayers [5] and virus imaging [6], provide basic knowledge about the contribution of individual components to the processes under consideration.

Motivated by the challenge of quantifying binding at early stages of infection, we have designed a setup to investigate binding kinetics between surface-immobilized virions and model membranes in the form of freely diffusing vesicles [Fig. 1(a)]. Individual fluorescent vesicles were tracked over time with total-internal-reflection fluorescence microscopy (TIRFM), enabling the vesicles in close proximity to the virus particles to be selectively illuminated, while the background fluorescence from vesicles in solution is efficiently suppressed. This means that, with the virus particles sufficiently separated on the surface, the lipid vesicles can be used to identify individual virus particles as shown in Fig. 1(b), while at the same time single association and dissociation events can be recorded under equilibrium conditions [7].

Although the concept is generic, we focus in this work on the norovirus genogroup II.4 Dijon strain [8-10] and its interaction with membranes containing the glycosphingolipids (GSLs) $H$ type 1 and Lewis $b$ [5(b)]. Norovirus is a nonenveloped RNA virus, $38 \mathrm{~nm}$ in diameter, with a symmetric icosahedral capsid assembled from 180 copies of the capsid protein arranged into 30 hexagonal facets [11] (Fig. S4 in Ref. [12]). Since there are no in vitro culture systems for human noroviruses, we employ self-assembled capsid proteins recombinantly expressed in insect cells, because such viruslike particles (VLPs) exhibit a morphology and binding characteristics similar to those of real viruses [13]. These VLPs recognize a variety of cell surface glycoconjugates [9], which are often involved in the first step of virion-cell interaction [14].

The VLPs were first bound to a supported 1-palmitoyl-2oleoyl-sn-glycero-3-phosphocholine lipid bilayer containing 5\% $\mathrm{H}$ type 1 as ligands [Fig. 1(a)]. The zwitterionic background lipids make the surface highly resistant to nonspecific protein and vesicle binding [15], while the included GSLs promote a firm and specific VLP attachment. Subsequent addition of GSL-containing phospholipid vesicles, each including $\sim 1000$ fluorescently labeled lipids, enables binding and dissociation kinetics to be measured by using TIRFM over a broad time regime (the details of assay preparation and imaging are in Ref. [12]).

The influence of vesicle size $(61 \pm 24$ or $163 \pm 87 \mathrm{~nm})$ and GSL content ( 2 or $5 \%$ ) on association and dissociation kinetics was explored by using $\mathrm{H}$ type 1 . In addition, $\mathrm{H}$ type 1 was replaced by Lewis $\mathrm{b}$ (which differs from 

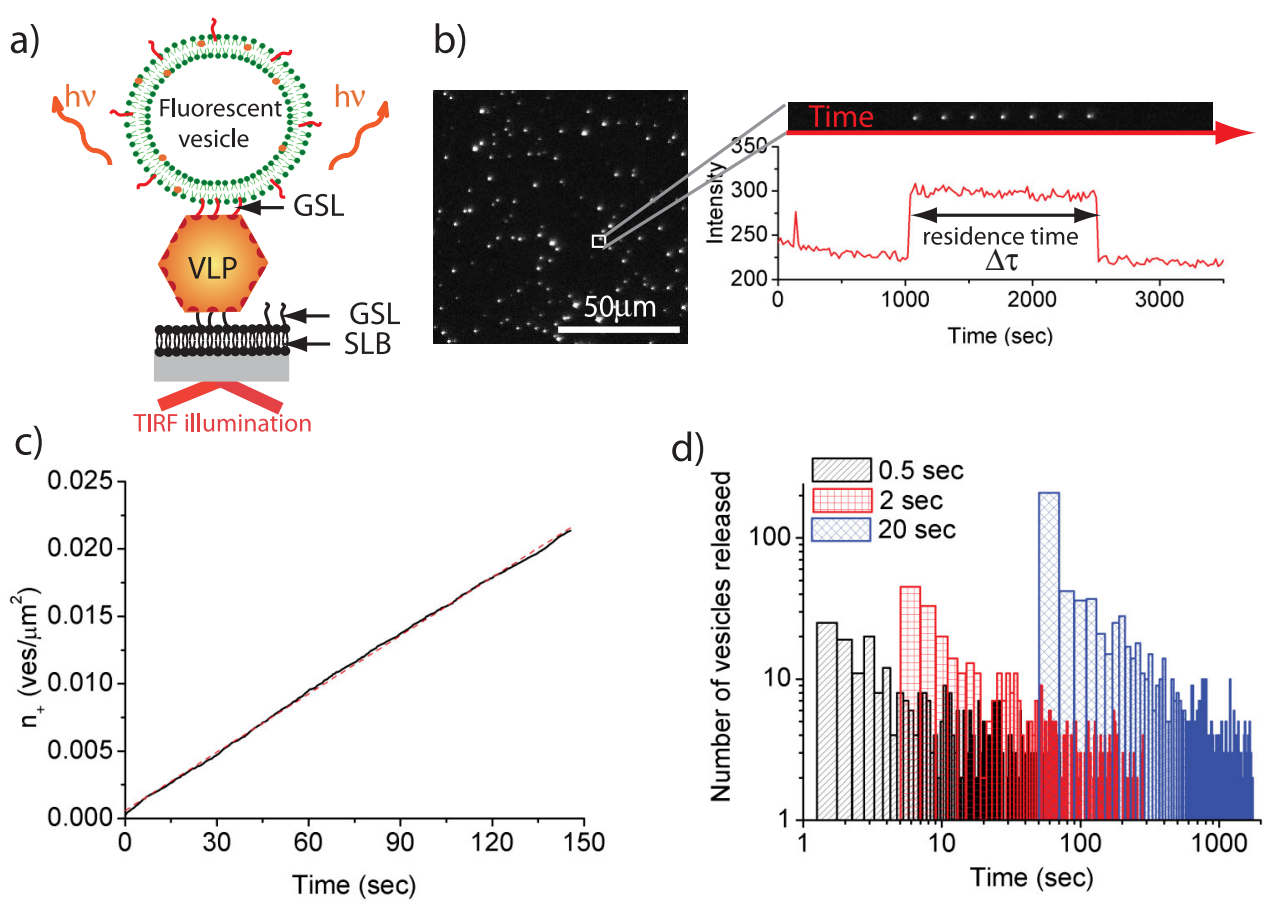

d)

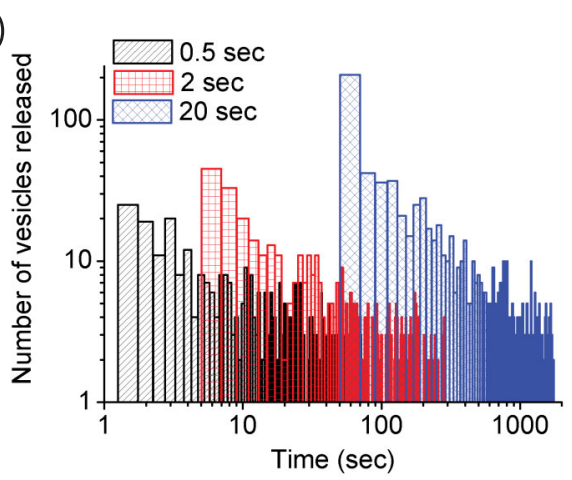

FIG. 1 (color online). Detection principle: (a) VLPs, immobilized on a supported lipid bilayer containing GSLs, interact with fluorescently labeled vesicles containing the GSL of interest. TIRF-based illumination is used to track surface-bound vesicles. (b) A typical TIRF image of surface-bound vesicles together with a kymograph and the intensity profile of a small image area containing a single vesicle. (c) Amount of newly arrived vesicles as a function of time. (d) Vesicle residence times for different time-lapse series. The data displayed here are for the $163 \mathrm{~nm}$ vesicles of type I.

$\mathrm{H}$ type 1 by the presence of one additional sugar unit), and the influence on binding kinetics was investigated. Therefore, the reported results correspond to vesicles of four types with size and GSL fractions: (I) $163 \mathrm{~nm}$ and 5\% $\mathrm{H}$ type 1, (II) $163 \mathrm{~nm}$ and 2\% $\mathrm{H}$ type 1, (III) $61 \mathrm{~nm}$ and 5\% $\mathrm{H}$ type 1, and (IV) $163 \mathrm{~nm}$ and 5\% Lewis b.

A key advantage of the concept employed here is that it allows studies of the kinetics not only in transient conditions but also in steady-state conditions, thus avoiding global diffusion limitations. Once binding equilibrium was reached, the association kinetics were investigated by recording the number of newly arrived vesicles, $n_{+}$, during the shortest times between frames $(0.5 \mathrm{~s}$ ). (To ensure that the system had time to relax towards equilibrium, the vesicles were added at least $30 \mathrm{~min}$ before the measurements were performed. The extension of this interval to $2 \mathrm{~h}$ did not change the results.) The linear increment of $n_{+}$vs time observed for vesicles of different types (Fig. 2) is consistent with steady-state conditions. The corresponding association rate can be represented as $d n_{+} / d t=k_{a} N_{\mathrm{VLP}} C_{\mathrm{ves}}$, where $k_{a}$ is the rate constant, $N_{\mathrm{VLP}}$ the VLP surface coverage, and $C_{\text {ves }}$ the concentration of vesicles in solution. We have used $C_{\text {ves }}=1.25$ and $0.35 \mathrm{p} M$ for the 61 and $163 \mathrm{~nm}$ vesicles, respectively. Based on binding experiments performed by using quartz crystal microbalance with dissipation monitoring, $N_{\mathrm{VLP}}$ was estimated to be $\sim 10 \mathrm{VLP} \mu \mathrm{m}^{-2}$. Employing these values and a linear fit $(y=a x)$ of the kinetics over the whole data range yields $k_{a}=(4.0 \pm 1.3) \times 10^{7},(1.1 \pm$ $0.4) \times 10^{7}$, and $(1.3 \pm 0.7) \times 10^{7} \mathrm{M}^{-1} \mathrm{~s}^{-1}$ for vesicles of types I, II, and III, respectively (the standard deviation was calculated from 4 measurements).

Although no global diffusion limitations are present, the elementary association events can be limited by vesicle diffusion (lateral diffusion of attached VLPs is not observed in our case); i.e., one should have $k_{a} \leq k_{\mathrm{D}} \simeq$ $2 \pi R_{c} D_{\text {ves }}$, where $R_{c}=19 \mathrm{~nm}$ is the VLP capsid radius and $D_{\text {ves }}$ is the vesicle diffusion coefficient [12]. Using the hydrodynamic $D_{\text {ves }}$ values 7.1 and $2.65 \mu \mathrm{m}^{2} / \mathrm{s}$ for 163 and $61 \mathrm{~nm}$ vesicles, respectively, we obtain $k_{\mathrm{D}} \simeq 2 \times 10^{8}$ for vesicles of types I and II and $5.3 \times 10^{8} \mathrm{M}^{-1} \mathrm{~s}^{-1}$ for vesicles of type III. In cases II and III, these values are appreciably larger than the measured ones, suggesting that the binding is kinetically limited. For vesicles of type $\mathrm{I}, k_{a}$ approaches the diffusion limit, which is attributed to their relative large size and higher GSL content. Using the Arrhenius representation, $k_{a}=k_{\mathrm{D}} \exp \left(-E_{a} / k_{\mathrm{B}} T\right)$, and the values of $k_{a}$ and $k_{\mathrm{D}}$ above, we have estimated the activation energy for association, $E_{a}=0.078$ and $0.10 \mathrm{eV}(1.8$ and $2.4 \mathrm{kcal} / \mathrm{mol})$, for vesicles of types II and III, respectively. These low values are physically reasonable, because the association is expected to start via formation and reorganization of one or two weak bonds. 


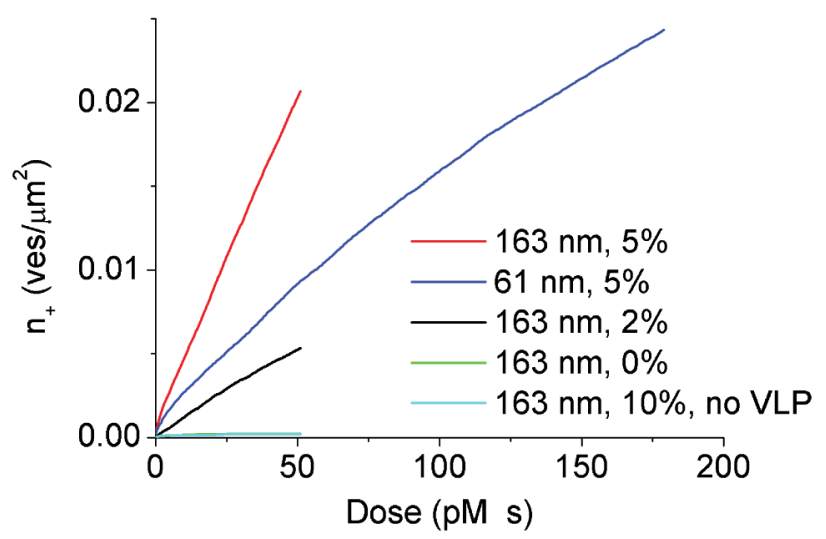

FIG. 2 (color online). Number of newly arrived vesicles as a function of dose $C_{\text {ves }} t$ for (from top to bottom) vesicles of types I (163 nm, 5\% H type 1, red curve), III (61 nm, 5\% H type 1, blue curve), and II (163 nm, 2\% $\mathrm{H}$ type 1, black curve). Control experiments were performed with fluorescent vesicles which did not contain GSL (163 nm, 0\%, green curve) or in the absence of VLPs (163 nm, 10\% H type 1, light blue curve). Both controls exhibit a hundredfold reduction in the association amount (the corresponding lines nearly coincide) compared to vesicles of type I. The measurements were executed with the time lapses of $0.5 \mathrm{~s}$.

VLP binding to GSL-containing lipid bilayers is governed by multiple weak interactions, which combined lead to high binding avidity. This makes information about dissociation kinetics challenging to acquire. Here, vesicle release was quantified by extracting the residence time of individual GSL-containing vesicles from time-lapse images [Fig. 1(d)] and displaying the number of vesicles still attached after a certain binding time (see, e.g., Fig. 3). To cover a broad range of residence times, time lapses of three different durations (150 s, $10 \mathrm{~min}$, and $1 \mathrm{~h}$ ) with different times between the frames $(0.5,2$, and $20 \mathrm{~s}$, respectively) were acquired. Guided by control experiments performed in the absence of VLPs or GSLs in the vesicles, a vesicle was considered as bound if it was present in at least three consecutive frames, setting thus the shortest residence time analyzed to 6 [Fig. 3(a)] or $1.5 \mathrm{~s} \mathrm{[Fig.} \mathrm{3(b)].}$

To further illustrate the potential of the method, the GSLs $H$ type 1 and Lewis b were compared. While the interaction patterns with these species were essentially identical when a conventional ensemble-averaging technique was used (Fig S3 in Ref. [12]), Fig. 3(a) shows that subtle differences in the interaction strength between the two GSLs can be resolved with single VLP sensitivity.

Interestingly, the release kinetics exhibit a distinct slowdown with increasing time manifested as a broad distribution in residence times [Fig. 1(d)]. To illustrate this feature over a wide time interval, it is helpful to use logarithmic coordinates. Specifically, we show $\ln [W(t) / n(0)]$ vs $\ln (t)$ (Fig. 4), where $W(t) \equiv-d n(t) / d t$ is the dissociation rate and $n(t)$ the number of vesicles bound (to minimize the noise, the data from the original time intervals were binned

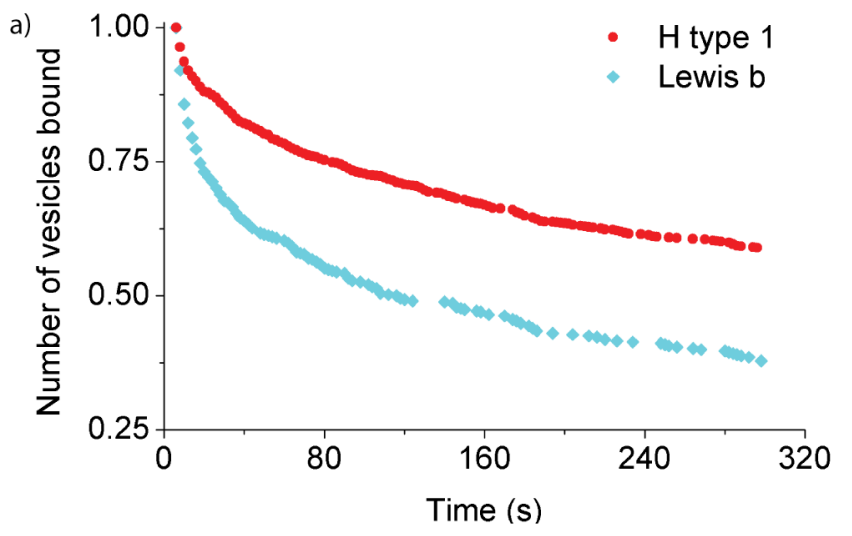

b)

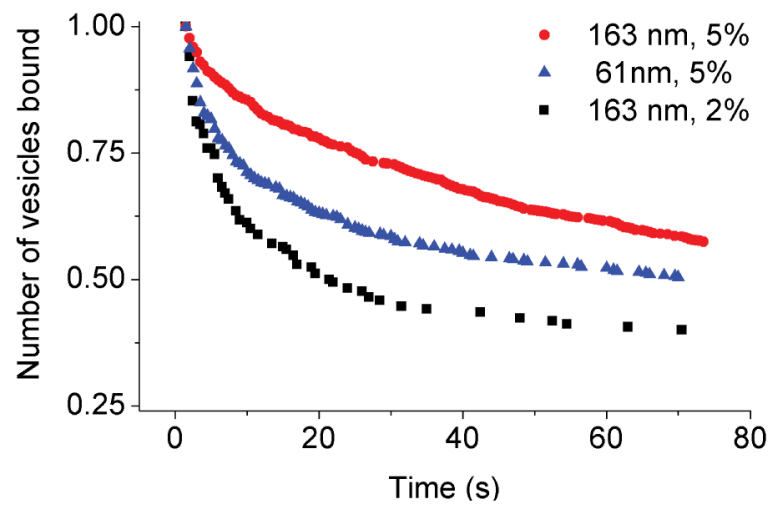

FIG. 3 (color online). Number of vesicles (arbitrary units) that are still bound as a function of time for (a) (from top to bottom) vesicles of types I (163 nm, 5\% $\mathrm{H}$ type 1 , red circles) and IV (163 nm, 5\% Lewis b, light blue diamonds) and (b) (from top to bottom) vesicles of types I (163 nm, 5\% H type 1, red circles), III (61 nm, 5\% H type 1, blue triangles), and II (163 nm, 2\% H type 1, black squares). The time lapses are (a) 0.5 and (b) $2 \mathrm{~s}$.

into larger time intervals; see Fig S2 in Ref. [12]). In all cases, the data exhibit a linear behavior with a characteristic slope fairly close to -1 over a very wide range of residence time (from 1 to about $10^{3} \mathrm{~s}$ ).

The dissociation rate constant for a vesicle of a given size depends on the corresponding activation energy $E$, determined primarily by the number of the GSL-capsidprotein bonds, and on the fraction of GSLs. To interpret the logarithmic kinetics, it is sufficient to focus on the activation energy, because energetics is the key factor in rate processes. Let us consider that, due to the vesicle-size distribution, $E$ is distributed in the range from $E_{\min }$ to $E_{\max }$. Assuming the preexponential factor for dissociation to be constant, we can represent the dissociation rate constant as $k(E)=k_{\max } \exp \left[-\left(E-E_{\min }\right) / k_{\mathrm{B}} T\right]$, where $k_{\max }$ is the maximum value. The number of bound species is given by $n(t)=n(0) \int_{E_{\min }}^{E_{\max }} f(E) \exp [-k(E) t] d E$, where $f(E)$ is the distribution of $E$. Combining these expressions with the simplest rectangular distribution $(f=1 / \Delta E$ with $\left.\Delta E \equiv E_{\max }-E_{\min }\right)$ and considering the kinetics to be studied in the time interval $1 / k_{\max } \ll t \ll 1 / k_{\min }$, we 


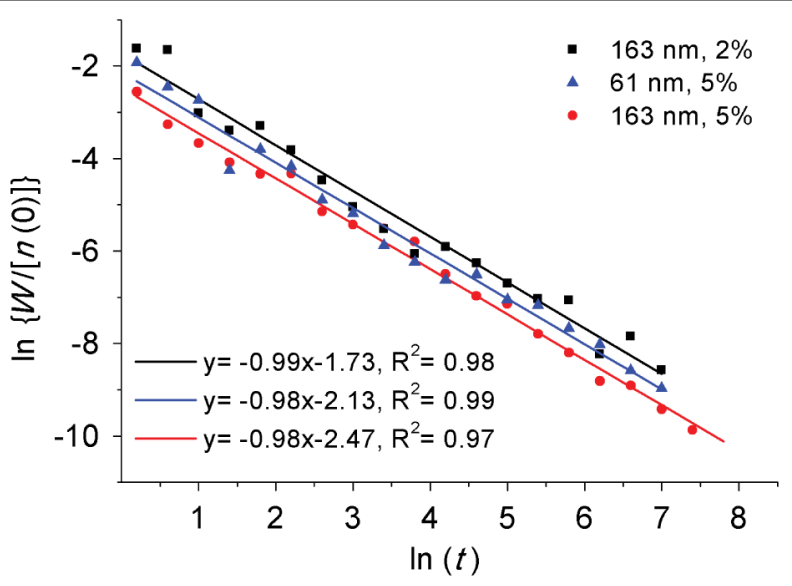

FIG. 4 (color online). Logarithmic plots of dissociation rate for (from top to bottom) vesicles of types II (163 nm, 2\% H type 1, black squares), III (61 nm, 5\% $\mathrm{H}$ type 1 , blue triangles), and I (163 nm, 5\% $\mathrm{H}$ type 1 , red circles) (time is in seconds). The data from the $0.5,2$, and $20 \mathrm{~s}$ time lapses have been merged and fitted linearly.

obtain $W(t)=-d n(t) / d t \simeq n(0) k_{\mathrm{B}} T /(t \Delta E)$ (for further details, see Ref. [12]). This expression, rewritten as $\ln [W(t) / n(0)]=\ln \left(k_{\mathrm{B}} T / \Delta E\right)-\ln (t)$, indicates, in good agreement with the experiment, that $\ln [W(t) / n(0)]$ linearly depends on $\ln (t)$ and the corresponding slope is -1 .

In natural sciences, the observations of logarithmic kinetics (as in Fig. 4) over a broad time range are not frequent. Such kinetics are usually associated with a broad distribution of one of the parameters controlling a rate process, in our case the activation energy of dissociation. As shown in Fig. 3(b), a decrease in vesicle size (from 163 to $61 \mathrm{~nm}$ ) or a decrease in ligand concentration (from 5\% to $2 \%$ ) results in an increase in the fraction of vesicles displaying shorter residence times. The binding energy between a GSL and capsid protein is weak $(\sim 0.1 \mathrm{eV})$, and firm binding occurs upon formation of several such bonds. In analogy with the initial stage of the virus entry by endocytosis [1], this process is expected to be facilitated by vesicle deformation allowing for an increase in the number of bonds. Deformation is more feasible for larger vesicles. For these reasons, the two likely factors behind the logarithmic kinetics of dissociation are (i) different GSL fractions in different vesicles and (ii) distribution of vesicle sizes. The role of the former factor seems to be minor, because the average number of GSLs per vesicle is large ( $\sim 12000, \sim 5000$, and $\sim 700$ for vesicles of types I, II, and III, respectively), and the fluctuations of this number are negligible. The latter factor appears to be more important, because the size distributions of extruded vesicles is broad (the full width at half maximum is close to the average; see Fig S1 in Ref. [12]).

In the case of logarithmic kinetics, the rate of a process at a given time $t$ is determined by the participants with the rate constant $k \simeq 1 / t$. In our context (Fig. 4), this means that we observe dissociation with rate constants in the range from about $10^{-3}$ to $1 \mathrm{~s}^{-1}$. Assuming for estimates that the preexponential factor for this process is normal $\left(10^{13} \mathrm{~s}^{-1}\right)$, and, taking into account that the experiments were performed at room temperature, we obtain that the dissociation activation energies corresponding to our experiments are in the range from 0.78 to $0.95 \mathrm{eV}$. Dividing these values by the energy of a single GSL-protein bond, we can estimate the number of GSLs participating in the association. As already noted, the single bond is weak. Using, e.g., $0.13 \mathrm{eV}(3 \mathrm{kcal} / \mathrm{mol})$, we obtain that the number of GSLs is between 6 and 8 . These values are in good agreement with estimates based on geometrical and structural considerations predicting that the number of bonds is between 6 and 12 [12].

In summary, the main outcome of our work is as follows.

(i) The single viral particle sensitivity, combined with the possibility to extract kinetic rate constants under stationary conditions, makes the TIRFM-based platform demonstrated in this work especially attractive to investigate simultaneously weak (few ligands) and strong (multiple ligands) interactions. It allows the study of heterogeneous binding systems over a broad range of residence times and without ensemble averaging. The simplicity of the assay, which does not require labeling of the viral particles, combined with low material consumption, makes it attractive in pharmaceutics, where methods allowing for screening of new compounds inhibiting virus binding and spreading are highly desirable. The single viral particle sensitivity makes it also competitive with conventional quantitative polymerase-chain-reaction-based diagnostics [8].

(ii) Our experiments with norovirus VLPs and vesicles containing glycosphingolipids indicate that the association rate constant of these species is lower than that expected for the diffusion-limited case. This finding in combination with the obtained information about the VLP-vesicle binding energy is useful for understanding the very first steps of interaction between virions and the host cell membrane, which was so far mainly focused on identification of the capsid binding sites responsible for this interaction [11].

(iii) The VLP-vesicle dissociation kinetics are observed to be logarithmic over a large range of time. This feature has been explained by multiple contacts between a vesicle and VLP and vesicle deformation. Such situations may occur at GSL-enriched cell-membrane regions with positive curvature [16]. This allows us to speculate that the logarithmic kinetics of the type observed may take place in vivo.

(iv) The dissociation kinetics also allow us to discriminate between two structurally similar GSLs, known from time-unresolved and semiquantitative assays to bind firmly to norovirus VLPs [17]. This observation is an additional argument in favor of potential applications of the technique for investigating constantly evolving virus strains. 
We acknowledge the Swiss National Science Foundation, VINNOVA, Swedish Foundation for Strategic Research, and Swedish Research Council for financial support and thank W. Nasir for constructing Fig. S4 presented in the Supplemental Materials.

*fredrik.hook@chalmers.se

[1] J. Mercer, M. Schelhaas, and A. Helenius, Annu. Rev. Biochem. 79, 803 (2010); A. J. Cann, Principles of Molecular Virology (Elsevier, Amsterdam, 2005).

[2] G. Seisenberger et al., Science 294, 1929 (2001); C. Brauchle et al., Chem. Phys. Chem. 3, 299 (2002).

[3] M. Lampe et al., Virology 360, 92 (2007); H. Ewers et al., Proc. Natl. Acad. Sci. U.S.A. 102, 15110 (2005); M. Lakadamyali et al., ibid. 100, 9280 (2003); D. Sivaraman et al., Trends Biotechnol. 29, 307 (2011).

[4] S. Manes et al., Nat. Rev. Immunol. 3, 557 (2003); D. Lingwood and K. Simons, Science 327, 46 (2010).

[5] (a) K. I. P. J. Hidari et al., Glycoconjugate J. 24, 583 (2007); (b) G.E. Rydell et al., Glycobiology 19, 1176 (2009).

[6] A. Bitler et al., Ultramicroscopy 110, 694 (2010); P. Kukura et al., Nat. Methods 6, 923 (2009); H. Ewers et al., Nano Lett. 7, 2263 (2007); F. A. Carneiro et al., Eur. Biophys. J. 35, 145 (2006).
[7] A. Gunnarsson et al., Nano Lett. 8, 183 (2008); Nucleic Acids Res. 37, e99 (2009).

[8] B. Nicollier-Jamot, V. Pico, P. Pothier, and Kohli, J. Clin. Microbiol. 41, 3901 (2003); M. M. Patel et al., J. Clin. Virol. 44, 1 (2009).

[9] G. E. Rydell, E. Kindberg, G. Larson, and L. Svensson, Rev. Med. Virol. (in press).

[10] Norovirus is the major causative agent of acute viral gastroenteritis worldwide causing yearly up to 200000 deaths of children [9]. It regularly leads to the shutdown of hospital units with immunocompromised patients due to its extremely high infectivity.

[11] B. V. V. Prasad et al., Science 286, 287 (1999); J.-M. Choi et al., Proc. Natl. Acad. Sci. U.S.A. 105, 9175 (2008).

[12] See Supplemental Material at http://link.aps.org/ supplemental/10.1103/PhysRevLett.107.188103 for detailed descriptions of materials and methods, derivations of analytical expressions, and a reasoning on the expected number of the VLP-vesicle bonds.

[13] X. Jiang et al., J. Virol. 66, 6527 (1992).

[14] M. Marsh and A. Helenius, Cell 124, 729 (2006).

[15] K. Glasmastar et al., J. Colloid Interface Sci. 246, 40 (2002).

[16] S. Sonnino et al., Glycobiology 17, 1R (2006).

[17] G. Rydell et al., Glycobiology 19, 309 (2009); J. Nilsson et al., Glycoconjugate J. 26, 1171 (2009). 